\title{
Sidewall lithography of micron-sized features in high-aspect-ratio meso-scale channels using a three-dimensional assembled mask
}

\author{
Chang-Hyeon $\mathrm{Ji}^{1,4^{*}+}$, Florian Herrault ${ }^{2,4^{*+}}$ and Mark G Allen ${ }^{3,4}$
}

\begin{abstract}
We report a fabrication technique for dense, detailed lithographic patterning on high-aspect-ratio vertical sidewalls, and illustrate its use through the fabrication of etched array of micro-dimples on the sidewalls of aluminum channels. A photoresist etch mask was spray-coated inside the channel, and patterned using a silicon-fabricated three-dimensional mask which includes a diffuser and a reflector to uniformly redistribute the vertically incident source light in the channel. All the lithographic processes from photoresist coating to development have been carried out without disassembling the channel. Arrays of $107.5 \mu \mathrm{m}$-deep circular dimples were isotropically etched onto the sidewalls of a $31 \mathrm{~mm}$-tall, $3.2 \mathrm{~mm}$-wide aluminum channel.
\end{abstract}

\section{Introduction}

In applications such as three-dimensional (3-D) electrical interconnect formation or surface texturing [1], the ability to fabricate micro-patterns on straight vertical sidewalls is very attractive, yet extremely challenging. Despite the recent advances in 3-D microfabrication technologies, little progress has been made in the development of a lithographic patterning process on 3-D surfaces, especially on vertical sidewalls. Unless the substrate is inclined or the direction of the incident light is deflected, direct exposure or patterning of the vertical sidewall is almost impossible and the level of difficulty or complexity rises if the sidewall is a part of a narrow or high-aspect-ratio channel. Techniques such as shadow mask deposition [2], inclined laser patterning [3], or metal transfer micromolding [4] have been investigated. Morishita et al. fabricated multi-height shadow mask to improve the pattern definition at the bottom and vertical sidewall of the trench compared to conventional planar shadow mask [2]. Rajaraman et al. used laser ablation process to partially scribe the conformally deposited polymer layer covering

\footnotetext{
* Correspondence: cji@ewha.ac.kr; florian@gatech.edu

${ }^{\dagger}$ Equal contributors

${ }^{1}$ Current address: Department of Electronics Engineering, Ewha Womans

University, Seoul 120-750, South Korea

${ }^{2}$ Current address: HRL Laboratories, 3011 Malibu Canyon Road, 90265 Malibu,

CA, USA

Full list of author information is available at the end of the article
}

the vertical sidewall of the 3-D metal electrode [3]. Zhao et al. used combination of multiple molding and metal deposition processes to pattern metal layer on the vertical sidewall of the structure [4]. Although these processes provide practical means to serve their needs, available pattern geometries and process flexibility are limited.

In this research, we report a new sidewall patterning technology based on a conventional photolithography process using spray-coated photoresist and silicon fabricated 3-D mask. In contrast to the previous sidewall patterning approaches, we have developed a direct patterning process by redirecting the highly directional light from the exposure tool. Analogous to the lithography process on a conventional two-dimensional surface where substrates are aligned and exposed subsequently, batch fabrication approach can be utilized in the sidewall patterning process using the proposed 3-D mask. As the channel measures $31 \mathrm{~mm}$-tall and $3.2 \mathrm{~mm}$-wide, exposure light incident on the narrow channel entrance on the top surface has been diffused and redistributed throughout the channel height by two reflecting surfaces. To our knowledge, direct patterning on the inner vertical sidewalls of a centimeter scale flow channel by lithographical means has not been attempted up to the present.

\section{Springer}

(c) $2014 \mathrm{Ji}$ et al.; licensee Springer. This is an Open Access article distributed under the terms of the Creative Commons Attribution License (http://creativecommons.org/licenses/by/4.0), which permits unrestricted use, distribution, and reproduction in any medium, provided the original work is properly cited. 


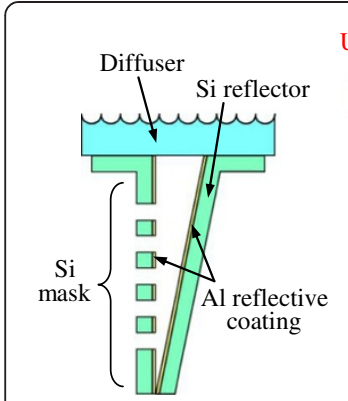

(a)

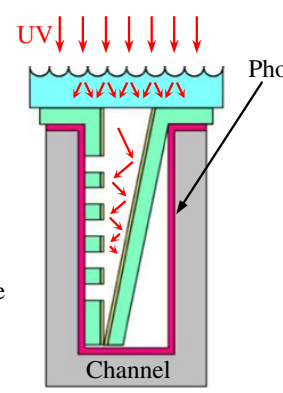

(b)

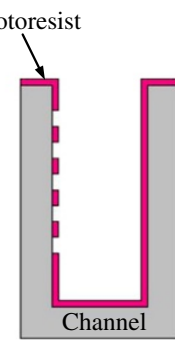

(c)
Figure 1 Schematics of the proposed approach; (a) 3-D mask, (b) exposure of photoresist-coated channel using 3-D mask, (c) result after development.

\section{Proposed method}

As shown in Figure 1(a), the 3-D mask consists of a vertically located mask plate which makes direct contact with the vertical sidewall to be patterned, an inclined reflector plate, and a diffuser. The diffuser plate is fabricated by forming micro-lens array by glass-etching process. Directional ultraviolet (UV) source light from an aligner or an exposure tool is first scattered by the diffuser plate and reflected across the patterned mask plate and reflector plate to fully reach the bottom of the mask plate while exposing the photoresist coated on the vertical sidewall (Figure 1(b)). The proposed approach is similar to the light-guide plate technology utilized in edge illuminated liquid crystal displays. Our technique differs from previously-reported concepts [2-4] as it benefits from the many advantages of photolithography process. Although application to smaller channels or vertical structures measuring millimeters to hundreds of microns require further investigation, the proposed approach can be utilized in various applications. As the process enables direct patterning of photoresist on the vertical part of the structure, theoretically any process available on the top surface is applicable to the sidewall. This includes applications such as surface features formation and sensor integration on vertical channel sidewalls.

\section{3-D mask design and fabrication}

The 3-D mask was fabricated by silicon deep-etching of a $500 \mu \mathrm{m}$-thick silicon substrate, and manual assembly process. Three silicon parts (mask, reflector, and field stop plates) have been fabricated by through-wafer etch using Bosch process, and have been assembled with a diffuser plate. The target patterns to be transferred to the vertical sidewall were formed on the silicon mask plate which was used as a dark field mask. As shown in Figure 2(b), the angle between the mask plate and reflector plate was $5.9^{\circ}$. The inner surfaces of the 3-D mask were coated with $1 \mu \mathrm{m}$-thick sputter-deposited aluminum before assembly for greater internal reflection. The micro-lenses on the diffuser plate were fabricated by etching a glass plate with 1:1:3 mixture of hydrofluoric acid, nitric acid, and deionized water using a chromium mask. A 2-dimensional array of $66 \mu$ m-diameter circles with $129 \mu$ m-pitch have been

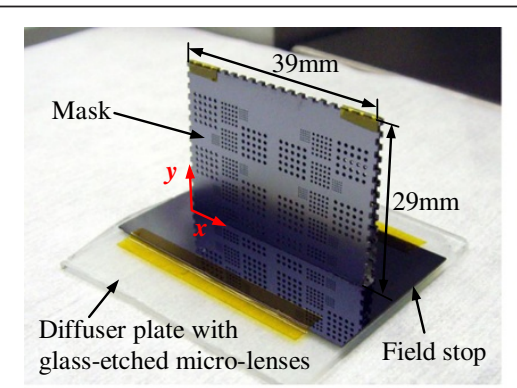

(a)

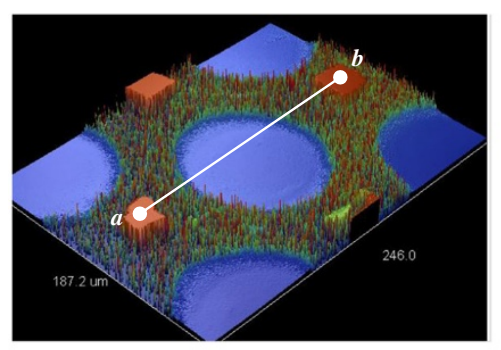

(c)

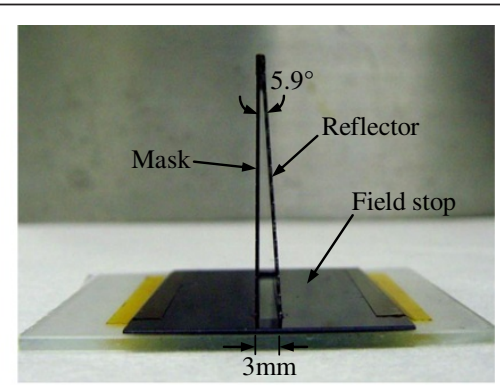

(b)

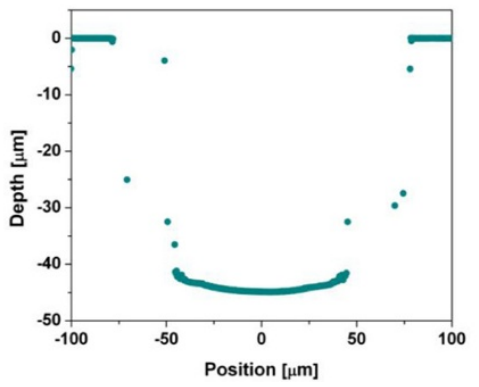

(d)

Figure 2 Fabricated 3-D mask; (a) perspective view, (b) side view, (c) 3-D white light interferometry profile of the glass-etched microlens formed on the diffuser plate, (d) 2-D profile along line $a b$ in (c). 


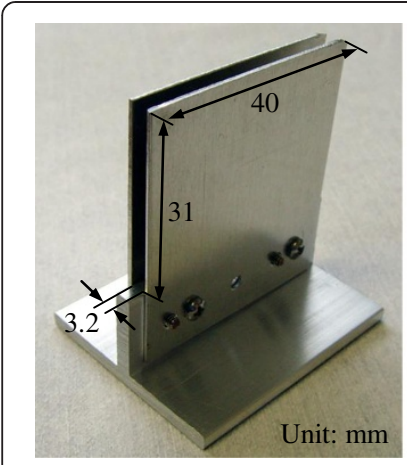

(a)

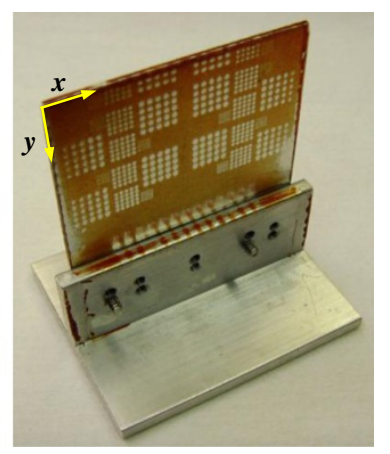

(b)
Figure 3 Assembled aluminum channel; (a) before lithography, (b) after lithography (one sidewall panel removed for post-processing analysis).

patterned on the chromium layer for the glass etching process. As shown in Figure 2(c), the etch depth was $46 \mu \mathrm{m}$ and the fill-factor of the micro-lens array was 95.5\%. A two-part epoxy-based adhesive has been used in the assembly process and polyimide tape has been used to provide additional structural rigidity.

\section{Findings}

Although extrusion is a common technique to fabricate high-aspect-ratio aluminum channels, our test structure consisted of a T-shaped base and two thin plates as sidewalls (Figure 3(a)). This assembly enabled post-processing analysis of the fabricated patterns by removing one plate. First, a positive-tone photoresist (AZ P4620, Clariant) was diluted by 1:1 with propylene glycol methyl ether acetate (PGMEA) and manually spray-coated inside the assembled channel using an off-the-shelf air-brush (Paasche Airbrush Company, model H3). Spray pressure was set to a constant value of 1.2 bar and the diluted photoresist was directly sprayed from the topside of the channel. Distance between the channel and spray head was approximately $5 \mathrm{~cm}$ and coating and baking on a $90^{\circ} \mathrm{C}$ hot plate for $1 \mathrm{mi}-$ nute was repeated 7 times. Estimated solids content of the diluted photoresist was $19 \%$ and the measured dispense rate of the air brush was approximately $1.5 \mathrm{ml} / \mathrm{min}$. The spray coating process has been detailed in [5]. The sidewalls were exposed using the 3-D mask and a UV light from a conventional mask aligner. Finally, the photoresist patterns were developed using air-brush spraying of a 3:1 mixture of deionized water and AZ $400 \mathrm{~K}$ developer (Figure 3(b)). Developer was directly sprayed into the channel from the top opening. After the development, deionized water was sprayed into the channel for rinsing and the sample was dried using nitrogen gun. Note that without the diffuser, the patterns were not defined in the upper region of the channel $(0<\mathrm{y}<17.4 \mathrm{~mm})$, confirming the micro-lenses' effectiveness (Figure 4). Due to the high directionality of the vertically incident UV light from the exposure tool and narrow angle between the reflector and mask, upper region of the vertical sidewall cannot be exposed properly without the aid of diffuser. As shown in Figure 4(a), only the bottom part of the sidewall has been exposed without the diffuser, which is a consequence of multiple reflection and diffraction inside the 3D mask. Microlens array acts as a diffuser to provide inclined reflector with incident UV rays having various directionalities, so that the reflected light can be incident on the vertical sidewall. In Figure 5, the 3-D plots highlight the process results as a function of lateral and vertical locations. Tested mask patterns ranged from 200 to $600 \mu \mathrm{m}$. Measured average pattern heights of the 600, 400, $200 \mu \mathrm{m}$ patterns were $938,666,312 \mu \mathrm{m}$, and average pattern widths were $815,582,259 \mu \mathrm{m}$, respectively. For the

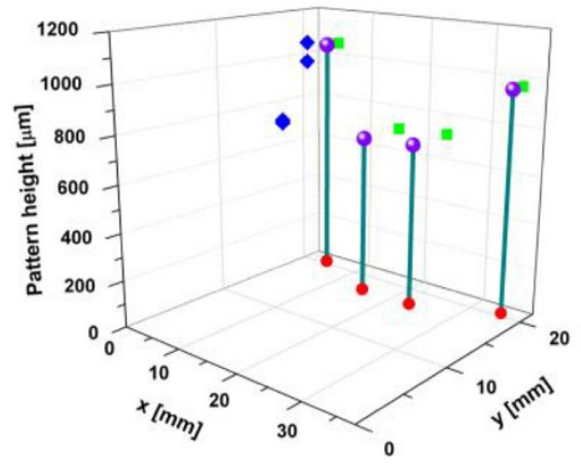

(a)

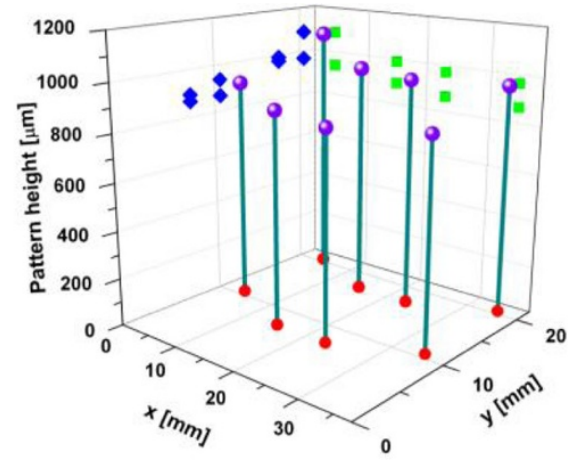

(b)

Figure 4 Exposure test results of spin-coated $1.4 \mu \mathrm{m}$-thick positive-tone photoresist (S1813, Shipley, $600 \mu \mathrm{m}$-diameter pattern, $\lambda=365 \mathrm{~nm}$, exposure dose: $7,000 \mathrm{~mJ} / \mathrm{cm}^{2}$, Purple dot represents the pattern height vs. pattern location on $x y$ plane. Blue diamond, green rectangle, and red circle represent pattern height vs. y position, pattern height vs. $x$ position, and pattern location on $x y$ plane, respectively.); (a) without diffuser, (b) with diffuser. 


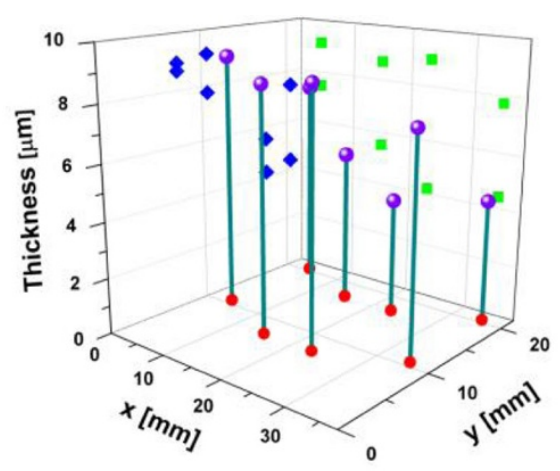

(a)

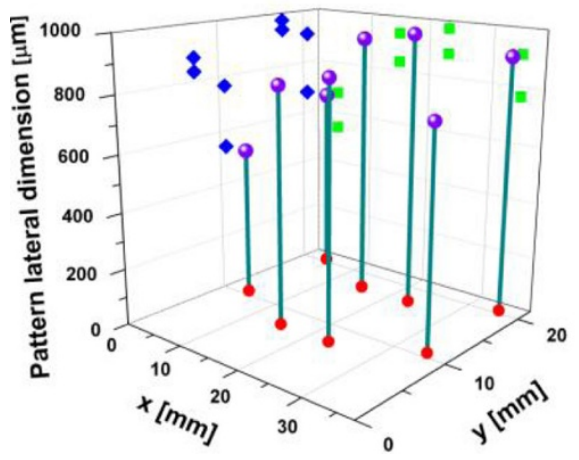

(c)

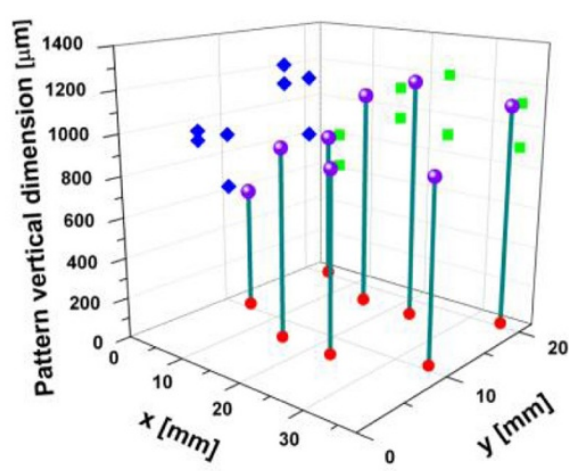

(b)

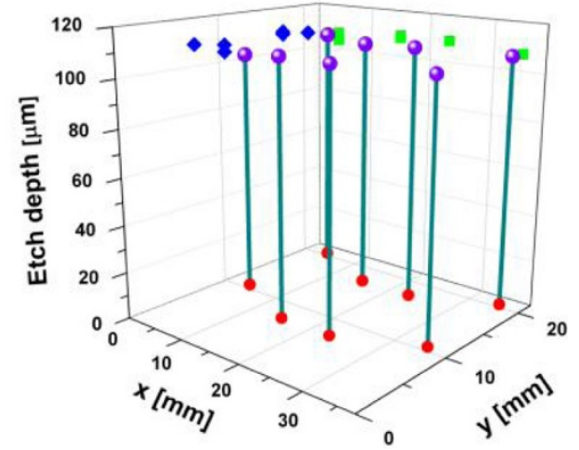

(d)

Figure 5 Patterning and etching results of $600 \mu \mathrm{m}$-diameter patterns as a function of pattern location $\left(\lambda=365 \mathrm{~nm}\right.$, dose: $8,550 \mathrm{~mJ} / \mathrm{cm}^{2}$, Purple dot represents the pattern height vs. pattern location on $x y$ plane. Blue diamond, green rectangle, and red circle represent pattern height vs. y position, pattern height vs. x position, and pattern location on xy plane, respectively.); (a) spray-coated photoresist thickness, (b) pattern vertical dimension, (c) pattern lateral dimension, (d) aluminum etch depth.

$600 \mu \mathrm{m}$-diameter patterns, measured pattern width ranged between 541-972 $\mu \mathrm{m}$ whereas the pattern height ranged between $613-1,196 \mu \mathrm{m}$. An average size increase of $56 \%$ was observed for the vertical dimension of the pattern and average shape distortion was 13\% (Figure 5(a)-(c)). The vertical dimensions were consistently larger than the lateral ones, which can be attributed to an imperfect mask-to-sidewall contact, and the direction of the light incident on the mask plate. Furthermore, the size variation of similar features throughout the channel height was estimated at $20 \%$. However, a strong correlation between the non-uniformity of the spray-coated photoresist thickness and the pattern size variation was observed. For testing purposes, pre-photoresist-coated

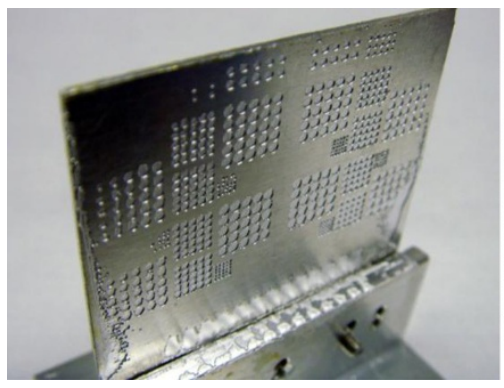

(a)

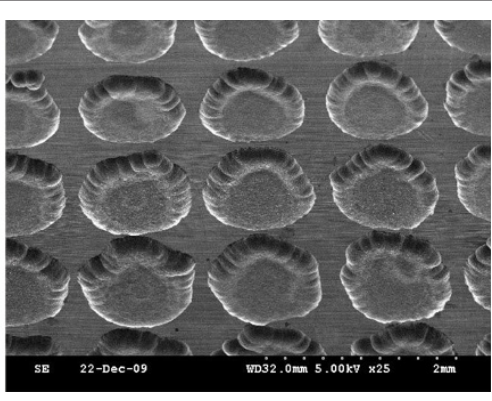

(b)

Figure 6 Fabricated dimples; (a) channel sidewall after Al etch and resist removal, (b) SEM image of an array of $600 \mu$ m-diameter dimples. 
sidewalls were assembled and exposed with the 3-D mask. The size variation of these photoresist patterns was reduced down to $7.8 \%$, indicating that an improved spray-coating process would yield better uniformity and greater control of the feature dimensions. Alternatives such as the use of electrodeposited photoresist may also improve the patterning result by improving the coating uniformity [6]. Also, improvement of 3D mask structure to prevent the leakage of incident light at the bottom and side edges can be achieved by leak-tight opaque sealing of silicon components or utilization of transparent polymer components covered with patterned metal layers.

The micro-dimples were formed on the channel sidewalls by wet etching the aluminum channel using the patterned photoresist as an etch mask. Outer surface of the assembled channel has been manually covered with a positive-tone photoresist (AZ P4620) and the whole structure was dipped in aluminum etchant (Aluminum etchant type A, Transene) at $40^{\circ} \mathrm{C}$. Measured etch depth of the $600 \mu \mathrm{m}$-diameter dimples was approximately $107.5 \mu \mathrm{m}$ with a standard deviation of $1.7 \mu \mathrm{m}$. Figure 6 shows one channel sidewall with micro-dimples and the associated scanning electron microscope (SEM) image.

\section{Conclusion}

This paper reports a direct lithography process on a vertical sidewall using a silicon deep-etched and assembled 3-D mask. To overcome the directionality of the UV source in conventional exposure tool, we have fabricated a 3-D silicon structure whose inner cavity mimics the lightguide plate used in edge illuminated liquid crystal displays. Sidewalls of a $31 \mathrm{~mm}$-deep and $3.2 \mathrm{~mm}$-wide flow channel have been patterned by exposing the spray coated photoresist with the fabricated 3-D mask. Using the patterned photoresist as the etch mask, arrays of $107.5 \mu$ m-deep circular dimples have been fabricated successfully. To our knowledge, this is the first direct lithography process to pattern the inner vertical sidewalls of a centimeter scale flow channel. The process can be utilized in various types of 3-D structure fabrication processes including surface feature generation and transducer formation on vertical sidewall of the channel or structure.

\section{Competing interests}

The authors declare that they have no competing interests.

\section{Authors' contributions}

$\mathrm{CHJ}$ and $\mathrm{FH}$ carried out the experiments, analyzed the experimental results, and drafted the manuscript. All the authors discussed the proposed process and experimental results. All the authors read and approved the final manuscript.

\section{Author details}

${ }^{1}$ Current address: Department of Electronics Engineering, Ewha Womans University, Seoul 120-750, South Korea. ${ }^{2}$ Current address: HRL Laboratories, 3011 Malibu Canyon Road, 90265 Malibu, CA, USA. ${ }^{3}$ Current address:

Department of Electrical and Systems Engineering, University of Pennsylvania, 200 South 33rd St., 19104-6314 Philadelphia, PA, USA. ${ }^{4}$ Georgia Institute of Technology, 791 Atlantic Dr., 30332 Atlanta, GA, USA.
Received: 16 April 2014 Accepted: 22 July 2014

Published online: 15 August 2014

\section{References}

1. Wei XJ, Joshi YK, Ligrani PM (2007) Numerical simulation of laminar flow and heat transfer inside a microchannel with one dimpled surface. J Electron Packag 129(1):63-70

2. Morishita S, Kim JH, Marty F, Li Y, Walton AJ, Mita Y (2009) A three-dimensional silicon shadowmask for patterning on trenches with vertical walls. Proc 15th Int Conf Solid-State Sensors, Actuators and Microsystems 1608-11

3. Rajaraman S, Choi SO, Shafer RH, Ross JD, Vukasinovic J, Choi Y, DeWeerth SP, Glezer A, Allen MG (2007) Microfabrication technologies for a coupled three-dimensional microelectrode, microfluidic array. J Micromech Microeng 17(1):163-71

4. Zhao Y, Yoon YK, Choi SO, Wu X, Liu Z, Allen MG (2009) Three dimensional metal pattern transfer for replica molded microstructures. Appl Phys Lett 94(2):023301

5. $\mathrm{Ji} \mathrm{CH}$, Herrault F, Allen MG (2008) A metallic buried interconnect process for through-wafer interconnection. J Micromech Microeng 18(10pp):085016

6. Pham NP, Boellaard E, Burghartz JN, Sarro PM (2004) Photoresist coating methods for the integration of novel 3-D RF microstructures.

J Microelectromech Syst 12:491-9

doi:10.1186/s40486-014-0006-7

Cite this article as: Ji et al.: Sidewall lithography of micron-sized features in high-aspect-ratio meso-scale channels using a three-dimensional assembled mask. Micro and Nano Systems Letters 2014 2:6.

\section{Submit your manuscript to a SpringerOpen ${ }^{\circ}$ journal and benefit from:}

- Convenient online submission

Rigorous peer review

- Immediate publication on acceptance

- Open access: articles freely available online

- High visibility within the field

- Retaining the copyright to your article

Submit your next manuscript at springeropen.com 